\title{
LIE GROUPS WHICH ADMIT FLAT LEFT INVARIANT METRICS
}

\author{
JOHN R. HERRING AND JOHN J. O'SULLIVAN
}

\begin{abstract}
Let $\boldsymbol{G}$ be a Lie group which admits a flat left invariant metric. We show that any nonflat left invariant metric on $G$ has conjugate points and we describe how some of the conjugate points arise.
\end{abstract}

Introduction. Milnor [6] has shown that a Lie group $G$ admits a flat left invariant metric if and only if the Lie algebra $g$ can be written an orthogonal direct sum $\mathfrak{b} \oplus \mathfrak{a}$ where $\mathfrak{b}$ is a commutative subalgebra, $\mathfrak{a}$ is a commutative ideal and $\operatorname{ad}_{B}$ is skew symmetric for every $B \in \mathfrak{b}$. The purpose of this paper is to prove the following.

THEOREM. If $G$ admits a flat left invariant metric, then any nonflat left invariant metric has conjugate points.

We first recall that, if $X$ and $Y$ are left invariant vector fields, the Riemannian connection $\nabla$ of a left invariant metric $\langle$, $\rangle$ satisfies

$$
\nabla_{X} Y=\frac{1}{2}\left\{\operatorname{ad}_{X} Y-\operatorname{ad}_{X}^{*} Y-\operatorname{ad}_{Y}^{*} X\right\}
$$

where * denotes transpose with respect to the inner product $\langle$, $\rangle$ (see [1, p. 64]). Let $e$ denote the identity element and $g$ an arbitrary element of $G$. If $\bar{Y}$ is a right invariant vector field, then $\bar{Y}$ satisfies

$$
\bar{Y}_{g}=L_{g^{*}}\left[\operatorname{Ad}\left(g^{-1}\right) \bar{Y}_{e}\right]
$$

We will not distinguish between elements of the Lie algebra and their left translates and will therefore write

$$
\bar{Y}_{g}=\operatorname{Ad}\left(g^{-1}\right) \bar{Y}_{e} .
$$

Let $E_{1}, \ldots, E_{n}$ be an orthonormal basis for $g$, let $X \in \mathfrak{g}$ and let $A$ denote the matrix of $\operatorname{ad}_{X}$ with respect to this basis. Suppose that $\bar{Y}=\sum_{i=1}^{n} f^{i} E_{i}$ and let $f$ be $f^{1}, \ldots, f^{n}$ written as a column vector. Along the one parameter group $c$ generated by $X$ we have

$$
\bar{Y}_{c(t)}=\operatorname{Ad}(\operatorname{Exp}(-t X)) \bar{Y}_{e}
$$

and so it follows by differentiation that, along $c, f$ is a solution of the ordinary differential equation

$$
\underset{f}{\dot{f}}+A f=0 .
$$

Received by the editors October 30, 1979 and, in revised form, April 28, 1980.

AMS (MOS) subject classifications (1970). Primary 53C30.

Key words and phrases. Lie group, flat left invariant metric, conjugate points.

() 1981 American Mathematical Society 0002-9939/81/0000-0269/\$02.00 
If $X$ is perpendicular to the commutator $g^{\prime}=[\mathfrak{g}, \mathfrak{g}]$, then $\operatorname{ad}_{Y}^{*} X=0$ for all $Y \in \mathfrak{g}$ and therefore it follows from (1) that the coefficient vector $f$ of a parallel vector field $\sum_{i=1}^{n} f^{i} E_{i}$ along $c$ is a solution of

$$
\underset{f}{\dot{f}}+\frac{1}{2}\left(A-A^{*}\right) \underset{\rightarrow}{f}=0 .
$$

Before proceeding with the proof of the theorem we need the following lemma.

LEMMA. Let $\mathfrak{a}^{c}$ be the complexification of $a$, then there exist $\mathbf{a d}_{\mathfrak{b}}$ invariant subspaces $a_{1}^{c}, a_{2}^{c}, \ldots, a_{l}^{c}$ of $a^{c}$ and real linear functionals $\lambda_{1}, \lambda_{2}, \lambda_{3}, \ldots, \lambda_{l}$ on $g$ such that

(i) $a^{c}=\bigoplus_{j=1}^{l} a_{j}^{c}$;

(ii) on each $\mathfrak{a}_{j}^{c}, \operatorname{ad}_{B}=i \lambda_{j}(B) I_{j}$

for every $B \in \mathfrak{b}$, where $I_{j}$ is the identity transformation on $\mathfrak{a}_{j}^{\mathfrak{c}}$.

Proof. The mapping $B \rightarrow \operatorname{ad}_{B}$ is a representation of the Abelian Lie algebra $\mathfrak{b}$ into $g l\left(a^{c}\right)$. Let $\rho_{1}, \rho_{2}, \ldots, \rho_{l}$ be the roots and $a_{1}^{c}, a_{2}^{c}, \ldots, a_{l}^{c}$ be the associated root spaces of the representation. Then $a^{c}=\bigoplus_{j=1}^{l} a_{j}^{c}$. For any root $\rho$ and any $B \in \mathfrak{b}$, $\rho(B)$ is an eigenvalue of a skew symmetric linear transformation and, therefore, must have zero real part.

Furthermore, $\left(\operatorname{ad}_{B}-\rho(B) I\right)^{k} U=0$ for $U \in a^{c}$ and $k>1$ if and only if

$$
\left(\operatorname{ad}_{B}-\rho(B) I\right) U=0 .
$$

It follows that each root $\rho_{j}$ is of the form $\rho_{j}=i \lambda_{j}$ where $\lambda_{j}$ is a real linear functional on $\mathfrak{b}$ and, on the corresponding root space, $\operatorname{ad}_{B}=i \lambda_{j}(B) I_{j}$. Q.E.D.

Proof of Theorem. Let $i \lambda$ be a root of ad: $\mathfrak{b} \rightarrow \mathfrak{g l}\left(\mathfrak{a}^{c}\right)$ and let $U \in \mathfrak{a}^{\mathfrak{c}}$ be a vector in the associated root space. Extend $\lambda$ to a real linear functional on all of $g$ by setting $\lambda=0$ on $a$. Since $a$ is an Abelian subalgebra it follows that for all $X \in \mathfrak{g}$

$$
\operatorname{ad}_{X}(U)=i \lambda(X) U \text {. }
$$

If Exp: $g \rightarrow G$ denotes the Lie algebra exponential mapping, it follows that for $g=\operatorname{Exp}(X)$

$$
\operatorname{Ad}(g) U=e^{i \lambda(X)} U .
$$

Without loss of generality, $G$ can be assumed to be connected, so we can write any $g \in G$ in the form

$$
g=\operatorname{Exp}\left(X_{1}\right) \operatorname{Exp}\left(X_{2}\right) \cdots \operatorname{Exp}\left(X_{r}\right)
$$

for some $X_{1}, X_{2}, X_{3}, \ldots, X_{r} \in g$, and so

$$
\operatorname{Ad}(g) U=\left\{\prod_{j=1}^{r} e^{i \lambda\left(X_{j}\right)}\right\} U=e^{i \lambda(X)} U
$$

where $X=\sum_{j=1}^{r} X_{j}$.

Suppose $U=Y_{1}+i Y_{2}$ and, for $i=1,2$, let $\bar{Y}_{i}$ be the right invariant vector field on $G$ which coincides with $Y_{i}$ at the identity element $e$. Since any right invariant vector field $\bar{Y}$ satisfies

$$
\bar{Y}_{g}=\operatorname{Ad}\left(g^{-1}\right) \bar{Y}_{e}
$$

it follows from (4) that, for any $g \in G$, we can find an element $X$ of $g$ such that

$$
\bar{Y}_{1}(g)=\operatorname{Re}\left\{e^{-i \lambda(X)} U\right\} \text { and } \bar{Y}_{2}(g)=\operatorname{Im}\left\{e^{-i \lambda(X)} U\right\} \text {. }
$$


Hence, for $i=1,2$, the length of $\bar{Y}_{i}$ is bounded above in any left invariant metric. If $\lambda \equiv 0, \bar{Y}_{i}$ has constant length. Otherwise $\exists Z \in g$ such that $\lambda(Z) \neq 0$ and then it follows from the periodicity of the sine and cosine functions that the length of $\bar{Y}_{i}$ attains an absolute maximum at some point along the one parameter subgroup generated by $Z$. By a result of O'Sullivan [7, Theorem B, p. 307] it follows that, if the length of a Killing vector field attains an absolute maximum, there are conjugate points unless the field is parallel. Hence there are conjugate points on $G$ unless $\bar{Y}_{1}$ and $\bar{Y}_{2}$ are both parallel vector fields. But since $i \lambda$ was any root of the representation ad: $\mathfrak{b} \rightarrow \mathfrak{g l}\left(\mathfrak{a}^{\mathfrak{c}}\right)$ and since $\mathfrak{a}^{\mathfrak{c}}$ is spanned by the root spaces, it follows that there are conjugate points on $G$ unless all right invariant vector fields $\bar{Y}$ with $\bar{Y}_{e} \in \mathfrak{a}$ are parallel.

Suppose all such vector fields are parallel in a left invariant metric $\langle$, $\rangle$. Let $X \in \mathfrak{a}^{\perp}$, the orthogonal complement of $\mathfrak{a}$ in $\mathfrak{g}$. Since $\mathfrak{a}$ is an ideal and $\mathfrak{b}$ is Abelian, it follows that the commutator $\mathfrak{g}^{\prime}=[\mathfrak{g}, \mathfrak{g}]$ is contained in $\mathfrak{a}$ and so $X$ is perpendicular to $g^{\prime}$. Therefore, if $Y \in \mathfrak{a}$, it follows from (2) and (3) that

$$
\operatorname{ad}_{X} Y=\frac{1}{2}\left(\operatorname{ad}_{X} Y-\operatorname{ad}_{X}^{*} Y\right)
$$

i.e.

$$
\operatorname{ad}_{X} Y=-\operatorname{ad}_{X}^{*} Y
$$

Since $a$ is an ideal, $\operatorname{ad}_{X}$ preserves $a$ and so, by (5), ad $d_{X}^{*}$ must also preserve $a$. This implies that $\operatorname{ad}_{x}$ preserves $a^{\perp}$, i.e.,

$$
\operatorname{ad}_{X}\left(\mathfrak{a}^{\perp}\right) \subset \mathfrak{a}^{\perp}
$$

Since $g^{\prime} \subset \mathfrak{a}$, we also have

$$
\operatorname{ad}_{x}\left(\mathfrak{a}^{\perp}\right) \subset \mathfrak{a} .
$$

Therefore $\operatorname{ad}_{X}\left(a^{\perp}\right)=0$ and it follows that $a^{\perp}$ is an Abelian subalgebra.

We have now shown that $g$ splits as an orthogonal direct sum $a \oplus a^{\perp}$ where $a$ is an Abelian ideal and $a^{\perp}$ is an Abelian subalgebra. Further, by (5), $\operatorname{ad}_{X}$ is skew symmetric for every $X \in \mathfrak{a}$. Hence, by Milnor [0], the metric $\langle$, $\rangle$ is flat. Q.E.D.

We will now give a partial description of the conjugate points arising in the nonflat metric. Using the notation of the proof, let $i \lambda$ be a root of ad: $a^{\perp} \rightarrow g l\left(a^{q}\right)$ and $a_{\lambda}^{c}$ be the root space for $i \lambda$. Extending $\lambda$ to all of $g$ by setting $\lambda(a)=0$, we have for $X \in \mathfrak{g}, U=Y_{1}+i Y_{2} \in \mathrm{a}_{\lambda}^{\mathrm{c}}$,

$$
\operatorname{ad}_{X} U=i \lambda(X) U
$$

If $\lambda \equiv 0$ on $\mathrm{g}$, then $Y_{1}$ and $Y_{2}$ are both in the center of $\mathrm{g}$. So by [3, Theorem 1], for any $a, b \in \mathbf{R}, Y=a Y_{1}+b Y_{2}$ is either parallel and, therefore, orthogonal to $\mathfrak{g}^{\prime}=[\mathfrak{g}, \mathfrak{g}]$, or the one parameter group $\operatorname{Exp}(t Y)$ is a geodesic with even order periodic sets of conjugate points.

If $\lambda \neq 0$, then any nonzero right invariant vector field $\bar{Y}$, defined by $\bar{Y}_{e}=a Y_{1}+$ $b Y_{2}, a, b \in \mathbf{R}$, is of bounded length and assumes maximal length at some point $g \in G$. Let $A_{\bar{Y}}$ be the $(1,1)$ tensor field defined by

and let $f=\frac{1}{2}\langle\bar{Y}, \bar{Y}\rangle$.

$$
A_{\bar{Y}} X=-\nabla_{X} \bar{Y}
$$


Since $\bar{Y}$ is a Killing vector field, it follows that $A_{\bar{Y}}$ is skew symmetric, that $\operatorname{grad} f=A_{\bar{Y}} \bar{Y}$ and that for any vector $Z$

$$
R(Z, \bar{Y}) \bar{Y}=-\nabla_{Z}\left(A_{\bar{Y}} \bar{Y}\right)-A_{Y}^{2}(Z)
$$

In particular,

$$
\begin{aligned}
\operatorname{Ric}(\bar{Y}, \bar{Y}) & =-\operatorname{div}\left(A_{\bar{Y}} \bar{Y}\right)-\operatorname{Trace}\left(A_{\bar{Y}}^{2}\right) \\
& =-\Delta f-\operatorname{Trace}\left(A_{\bar{Y}}^{2}\right)
\end{aligned}
$$

where $\Delta$ denotes the Laplacian. (See [5, p. $248 \mathrm{ff}]$.)

Furthermore, the integral curve $c$ of $\bar{Y}$ through $g$ is a geodesic. Since $f$ is constant and maximal along $c$, it follows from (6) and (7) that $\operatorname{Ric}(\dot{c}, \dot{c})>0$ unless the restricted curvature tensor $R_{c}$, defined by $R_{c}(Z)=R(Z, \dot{c}) \dot{c}$ for $Z \in T_{c}(G)$, vanishes. By [2], there are conjugate points on a geodesic with positive Ricci curvature. If $Y_{0}=\left(L_{g-1}\right)_{*} \bar{Y}_{g}$, then $c_{0}=L_{g-1} c$, the one parameter group generated by $Y_{0}$, is a geodesic with conjugate points unless $R_{c_{0}}$ vanishes.

REMARK. Any compact manifold is finitely covered by the torus. Therefore if $G$ has a discrete subgroup $\Gamma$ such that the quotient $G / \Gamma$ is compact, one can choose $\Gamma$ so that the quotient is a torus. Any left invariant metric on $G$ would then produce a Riemannian metric on the torus with the same curvature properties and the same Jacobi fields. It is a consequence of our theorem that any such metrics on the torus must have conjugate points if they are not flat. In [4], E. Hopf showed that all nonflat metrics on the two dimensional torus have conjugate points, but the question remains open in higher dimensions.

\section{REFERENCES}

1. J. Cheeger and D. G. Ebin, Comparison theorems in riemannian property, North-Holland, Amsterdam, 1975.

2. D. Gromoll and W. Meyer, On complete open manifolds of pasitive curvature, Ann. of Math. (2) 90 (1969), 75-90.

3. J. R. Herring and J. J. O'Sullivan, The Jacobi equation on Lie groups with left invariant metrics (to appear).

4. E. Hopf, Closed surfaces without conjugate points, Proc. Nat. Acad. Sci. U.S.A. 34 (1948), 47-51.

5. S. Kobayashi and K. Nomizu, Foundations of differential geometry. Vol. 1, Interscience, New York, 1962.

6. J. Milnor, Curvature of left invariant metrics on Lie groups, Advances in Math. 21 (1976), 293-329.

7. J. J. O'Sullivan, Manifolds without conjugate points, Math. Ann. 210 (1974), 295-311.

Department of Mathematics, Pennsylvania State University, University Park, Pennsylvania 16802

Current address (J. R. Herring): 1815 LaSalle Place, Severn, Maryland 21144

Current address (J. J. O'Sullivan): 1009 Frederick Street, Vienna, Virginia 22180 